\title{
Effects of the combined use of a scissor-type knife and traction clip on endoscopic submucosal dissection of colorectal tumors: a propensity score-matched analysis $\square$
}

다 (i) $(-)$

\author{
Authors \\ Ken Inoue ${ }^{1}$, Naohisa Yoshida ${ }^{1}$, Osamu Dohi ${ }^{1}$, Satoshi Sugino ${ }^{1}$, Shinya Matsumura ${ }^{1}$, Hiroaki Kitae ${ }^{1}$, Ritsu Yasuda $^{2}$, \\ Takahiro Nakano $^{3}$, Kei Terasaki ${ }^{4}$, Ryohei Hirose ${ }^{1}$, Yuji Naito ${ }^{1}$, Takaaki Murakami ${ }^{5}$, Yutaka Inada ${ }^{3}$, Kiyoshi Ogiso $^{6}$, \\ Yukiko Morinaga7 $^{7}$, Mitsuo Kishimoto ${ }^{7}$, Itoh Yoshito ${ }^{1}$
}

Institutions

1 Department of Molecular Gastroenterology and Hepatology, Kyoto Prefectural University of Medicine, Kyoto, Japan

2 Department of Gastroenterology, Nara City Hospital, Nara, Japan

3 Department of Gastroenterology and Hepatology, Japanese Red Cross Society Kyoto Daiichi Hospital, Kyoto, Japan

4 Department of Gastroenterology and Hepatology, Saiseikai Suita Hospital, Suita-City, Japan

5 Department of Gastroenterology, Aiseikai Yamashina Hospital, Kyoto, Japan

6 Department of Gastroenterology, Osaka General Hospital of West Japan Railway Company, Osaka, Japan

7 Department of Surgical Pathology, Kyoto Prefectural University of Medicine, Graduate School of Medical Science, Kyoto, Japan

submitted 23.11.2020

accepted after revision 2.3.2021

Bibliography

Endosc Int Open 2021; 09: E1617-E1626

DOI 10.1055/a-1535-0786

ISSN 2364-3722

(c) 2021. The Author(s).

This is an open access article published by Thieme under the terms of the Creative Commons Attribution-NonDerivative-NonCommercial License, permitting copying and reproduction so long as the original work is given appropriate credit. Contents may not be used for commercial purposes, or adapted, remixed, transformed or built upon. (https://creativecommons.org/licenses/by-nc-nd/4.0/)

Georg Thieme Verlag KG, Rüdigerstraße 14,

70469 Stuttgart, Germany

Corresponding author

Ken Inoue, MD, PhD, Department of Molecular

Gastroenterology and Hepatology, Kyoto Prefectural

University of Medicine, Graduate School of Medical Science,

465 Kajii-cho, Kawaramachi-Hirokoji, Kamigyo-ku, Kyoto 602

8566, Japan
Fax: +81-75-251-0710

keninoue71@koto.kpu-m.ac.jp

\section{ABSTRACT}

Background and study aims This study evaluated the technical aspects of colorectal endoscopic submucosal dissection (ESD) with the Clutch Cutter (CC) (Fujifilm Co., Tokyo, Japan), a scissor-type knife, and the S-O clip (SO) as a traction clip, and compared the safety and efficacy to ESD using a needle-type knife.

Patients and methods This was a single-center retrospective study. In Study 1, we evaluated 125 ESD patients: 60 using the SO and CC (SO group) and 65 using the CC (CC group). In Study 2, we evaluated 185 ESD patients: the CC group $(\mathrm{N}=65)$ and 120 using the Flush knife BT-S (Flush group) (Fujifilm Co., Tokyo, Japan). In both studies, the clinicopathological features and therapeutic outcomes were compared using a propensity score-matched analysis.

Results In 36 pairs of matched patients in Study 1, the rates of en bloc resection, $\mathrm{R} 0$ resection, perforation, and postoperative bleeding (POB) were $97.2 \%, 88.9 \%, 2.8 \%$, and $0 \%$, respectively, for the SO group and $100 \%, 91.7 \%$, $0 \%$, and $0 \%$ for the CC group (not significant). The mean procedure time for the SO group among less-experienced endoscopists was significantly shorter than in the CC group (42 vs. 65 minutes, $P=0.036$ ). In 49 pairs of matched patients in Study 2, the rates of en bloc resection, $\mathrm{R} 0$ resection, perforation, and $\mathrm{POB}$ were $100 \%, 95.8 \%, 0 \%$, and $0 \%$, respectively, for the CC group and $98.0 \%, 95.8 \%, 0 \%$, and $2.0 \%$ for the Flush group (not significant). The mean procedure time in the CC group among less-experienced endoscopists was significantly shorter than in the Flush group (52 vs. 67 minutes, $P=0.038$ ).

Conclusions CC and the combined use of CC and SO reduced colorectal ESD procedure time among less-experienced endoscopists. 


\section{Introduction}

Endoscopic submucosal dissection (ESD) to treat colorectal cancer has an excellent curative resection rate. However, technical difficulties and high complication rates have limited its use worldwide. During ESD for colorectal cancer using a conventional needle-type knife, problems such as unintentional incisions, perforation and bleeding may be encountered.

Two kinds of scissor-type knives are available on the market: the Clutch Cutter (CC) (Fujifilm Co., Tokyo, Japan) and SB knife (Sumitomo Bakelite, Tokyo, Japan) [1,2]. Previous studies have reported that these scissor-type knives reduce the technical difficulty of gastrointestinal ESD [2,3]. Some have found that ESD using the CC is technically more efficient for resecting early gastrointestinal tumors than that using conventional needletype knives [4-8]. However, other reports have shown that colorectal ESD using a scissor-type knife requires a longer mean procedure time than that with conventional knives [1, 4]. Other reports found no marked difference in the median procedure time between scissor-type knife and conventional knives [9]. The recently developed traction device called the SO clip (Zeon Medical Co., Ltd., Tokyo, Japan) has reportedly helped reduce the procedure time with needle-type knives $[10,11]$.

The data regarding the efficacy and safety concerning the impact of the combined use of a scissor-type knife and traction device on the median procedure time for colorectal ESD are limited [12]. Therefore, in the present study, we compared the efficacy of the combined use of the CC and S-O clip with that of the $\mathrm{CC}$ alone. We also compared the efficacy, safety and procedure time of the $C C$ with that of a conventional needle-type knife for colorectal ESD.

\section{Patients and methods}

\section{Study design and patients}

A single-center retrospective study was conducted in the Department of Molecular Gastroenterology and Hepatology, Kyoto Prefectural University of Medicine. We analyzed 310 consecutive patients who received ESD for colorectal tumors from April 2015 and April 2018 in our center and conducted two studies on the CC as Studies 1 and 2 ( $\mathbf{F i g} .1$ ). In Study 1 to analyze the comparison between CC and CC with the S-O clip, a total of 60 consecutive patients who underwent ESD between May 2018 and December 2018 using the CC with the S-O clip (SO group) were included. A total of 65 consecutive patients who underwent ESD between May 2017 and April 2018 using the CC (CC group) were included. In Study 2 to analyze the comparison between CC and Flush knife, 120 patients who underwent colorectal ESD using the Flush knife supported by the CC between April 2015 and April 2017 (Flush group) were included compared to CC group. The indications for ESD included: (1) tumors that could not be resected using endoscopic mucosal resection and; (2) tumors diagnosed as intramucosal cancer (Tis), high-grade dysplasia or part of T1a cancer using magnifying endoscopic examinations, such as pit pattern observation, narrow-band imaging, flexible spectral imaging color enhancement and blue laser imaging.

Regarding the timeline for each group, the S-O clip with CC has been routinely used for colorectal ESD since May 2018 ( $\triangleright$ Fig.1). Before then, colorectal ESD was performed using only the CC. The CC has been routinely used for colorectal ESD since May 2017. Before then, colorectal ESD was performed mainly with the Flush knife supported by the CC from April 2015.

All patients provided their written informed consent to undergo ESD. This study was approved by the Ethical Review Com-

\section{0 consecutive patients receiving colorectal endoscopic submucosal dissection April 2015 - April 2018}

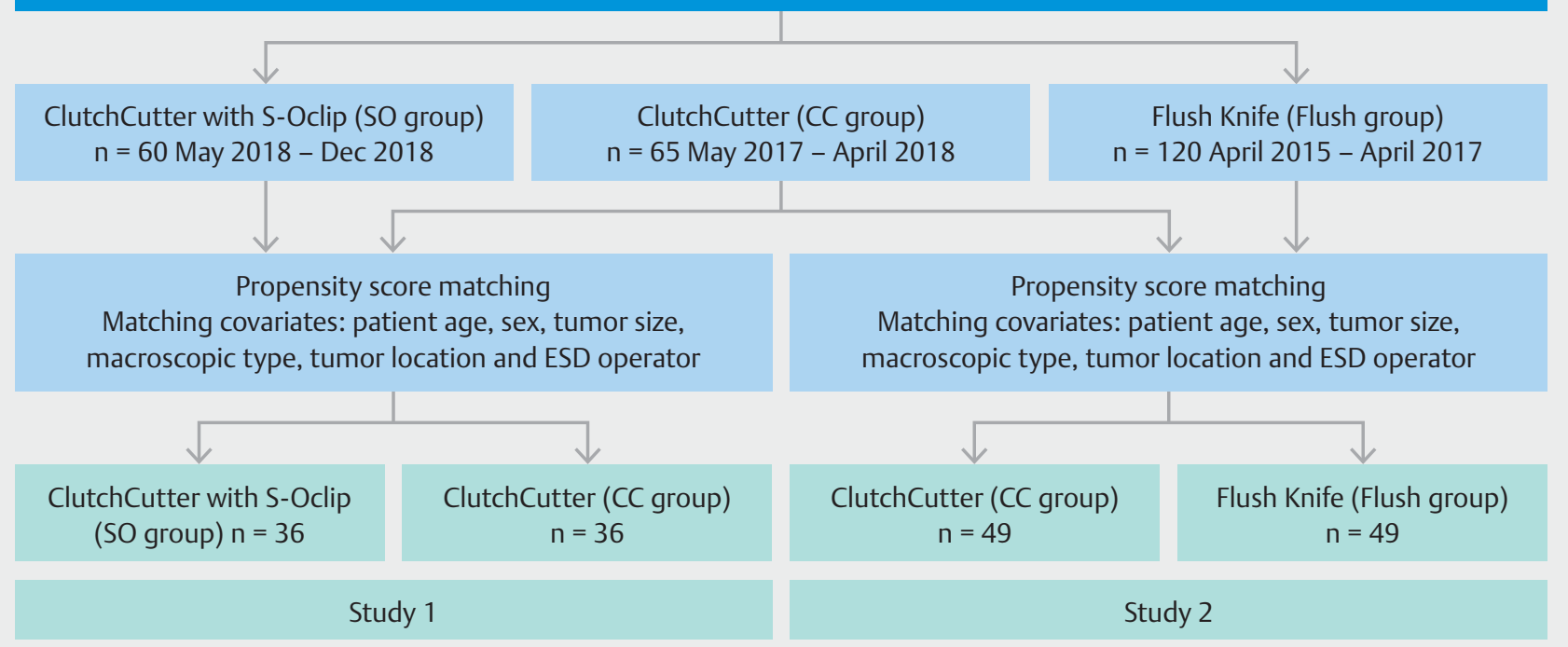

Fig. 1 Flowchart of Study 1 and Study 2.CC, Clutch Cutter; ESD, endoscopic submucosal dissection. 
mittee of the Kyoto Prefectural University of Medicine and carried out in accordance with the Helsinki Declaration of the World Medical Association.

The primary endpoint for the study was the procedure time and rates of en bloc resection and R0 resection in Study 1 and Study 2 . Secondary endpoints were the self-completion rate by the less-experienced endoscopists, the semi-self completion rate by the less-experienced endoscopists, the perforation rate and postoperative bleeding in each study. The procedure time was calculated as the time from the beginning of the local injection to the end of the procedure (completion of en bloc resection). Self-completion was defined as completion of ESD by the less-experienced endoscopists themselves without the procedure being taken over by their supervisors. Semi-self completion rate was defined as completion of ESD by the less-experienced endoscopists with the procedure being taken over by their supervisors within a quarter of the procedure time. All procedures were recorded on a hard disk drive for the evaluation of parameters.

\section{Endoscopic system, device and setting}

All colorectal ESD procedures were carried out with ECL600ZP or ECL600ZP7 colonoscopes and the LASEREO endoscopic system (Fujifilm Co.). A transparent hood (Elastic touch; Top Corporation, Tokyo, Japan) was attached to the tip of the scope. ESD procedures were performed while patients were under sedation using midazolam (Dormicum; Astellas Pharma, Tokyo, Japan) with pentazocine (Pentazin; Sankyo Pharmaceuticals, Tokyo, Japan). All procedures were done with a carbon dioxide insufflation system. Sodium hyaluronate (MucoUp; Boston Scientific, Tokyo, Japan) was used with a small amount of indigo carmine as an injection solution to achieve long-lasting high submucosal elevation. With respect to bowel preparation, patients consumed a low-residue diet and took sodium picosulfate $10 \mathrm{~mL} 1$ day before the examination. Furthermore, beginning in 2014 , all patients also received $1.0 \mathrm{~L}$ of a highly concentrated polyethylene glycol solution with ascorbic acid (MoviPrep; Ajinomoto Pharma Co., Ltd., Tokyo, Japan) in the morning on the day of the examination, as described in our previous report [13].

The CC is a commercially available scissor-type electrosurgical knife. It can grasp and cut target tissue using an electrosurgical current. The CC with a 3.5-mm-long serrated cutting edge and the VIO300D (ERBE, Tubingen, Germany) were used for colorectal ESD procedures between May 2017 and December 2018 (CC and SO groups). The S-O clip (Zeon Medical Co.), which consists of a spring and is connected to a nylon loop, was used for colorectal ESD procedures between April 2018 and December 2018.

Two expert endoscopists (N.Y. and K.I.) and five less-experienced endoscopists (R.H., T.M., R.Y., S.M. and H.K.) performed ESD for colorectal tumor. The expert endoscopist (N.Y.) performed ESD during all three periods. The expert endoscopist (K.I.) performed ESD during the third period (SO group). The less-experienced endoscopist (R.H.) performed ESD during the first period (Flush group). The less-experienced endoscopist (T.M.) performed ESD during the second period (CC group).
The less-experienced endoscopists (R.Y., S.M. and H.K.) performed ESD during the third period (SO group). The experts had experience performing $\geq 100$ colorectal ESD procedures using the Flush knife and $\geq 100$ colorectal ESD procedures using the CC. All less-experienced endoscopists had experience with $<20$ colorectal ESD procedures using the Flush knife or the CC at the beginning of their periods. Before they began performing ESD using the CC, less-experienced endoscopists observed ESD procedures performed by an expert for 1 month. All procedures by less-experienced endoscopists were performed under the supervision of experts (N.Y. or K.I.). Less-experienced endoscopists were changed to supervisors in the following cases: the estimated procedure time for mucosal incision and submucosal dissection was expected to exceed 60 minutes or there was uncontrollable hemorrhaging or perforation. Once operators changed to the supervisor, the ESD procedure was performed by the less-experienced endoscopists again if the supervisor was able to bring the issue (e.g. hemorrhaging) under control.

\section{ESD procedure using the $\mathrm{CC}$ and S-O clip}

The method using the $\mathrm{CC}$ and S-O clip has already been described in detail ( $>$ Fig. 2, > Fig. 3, > Video 1) [14]. In brief, after sufficient hyaluronic acid injection, a full circumferential mucosal incision using the CC was performed in Endocut I mode (Effect 1 , duration 4 , interval 1) from the oral side. To prevent knife slippage and incise mucosa and submucosa enough, we used Endocut I with open-style CC. This open-style CC method should be standard technique only with enough injection into submucosal layer. This method may induce perforation without enough injection. Submucosal dissection was performed with the combination of forced coagulation mode (Effect 2, 30 W) and Endocut I mode after a full circumferential incision. In the SO group, after full circumferential mucosal incision, the $\mathrm{S}-\mathrm{O}$ clip was attached to the proximal edge of the lesions. Then, the second clip was used to hook the loop of the S-O clip and fasten the loop to anal to the lesion and on the opposite colonic wall. In the SO group, only full circumferential mucosal incision of sufficient depth without trimming is enough for dissection. The open-style CC method was useful to have sufficient depth mucosal incision. All of these steps were performed with the same maneuver: (1) grasping the target tissue; (2) pulling the grasped tissue up (separation of the grasped tissue from the appropriate underlying muscle layer); and (3) cutting the grasped tissue (or coagulating the blood vessel) $[1,2,5,14]$. All of these steps are comparable to techniques used with a biopsy or coagulating forceps. Precoagulation of visible blood vessels using the $\mathrm{CC}$ is useful for preventing intraoperative bleeding. The vessel is grasped using the CC, and an electrical discharge is briefly performed with either forced coagulation (Effect 2, 30W) or soft coagulation (Effect 4, $100 \mathrm{~W}$ ). In the setting of active bleeding, hemostasis during ESD can be achieved with the CC by applying mechanical pressure by closing the blades around the target lesion followed by coagulation (soft coagulation mode, Effect 4, 100 W). 

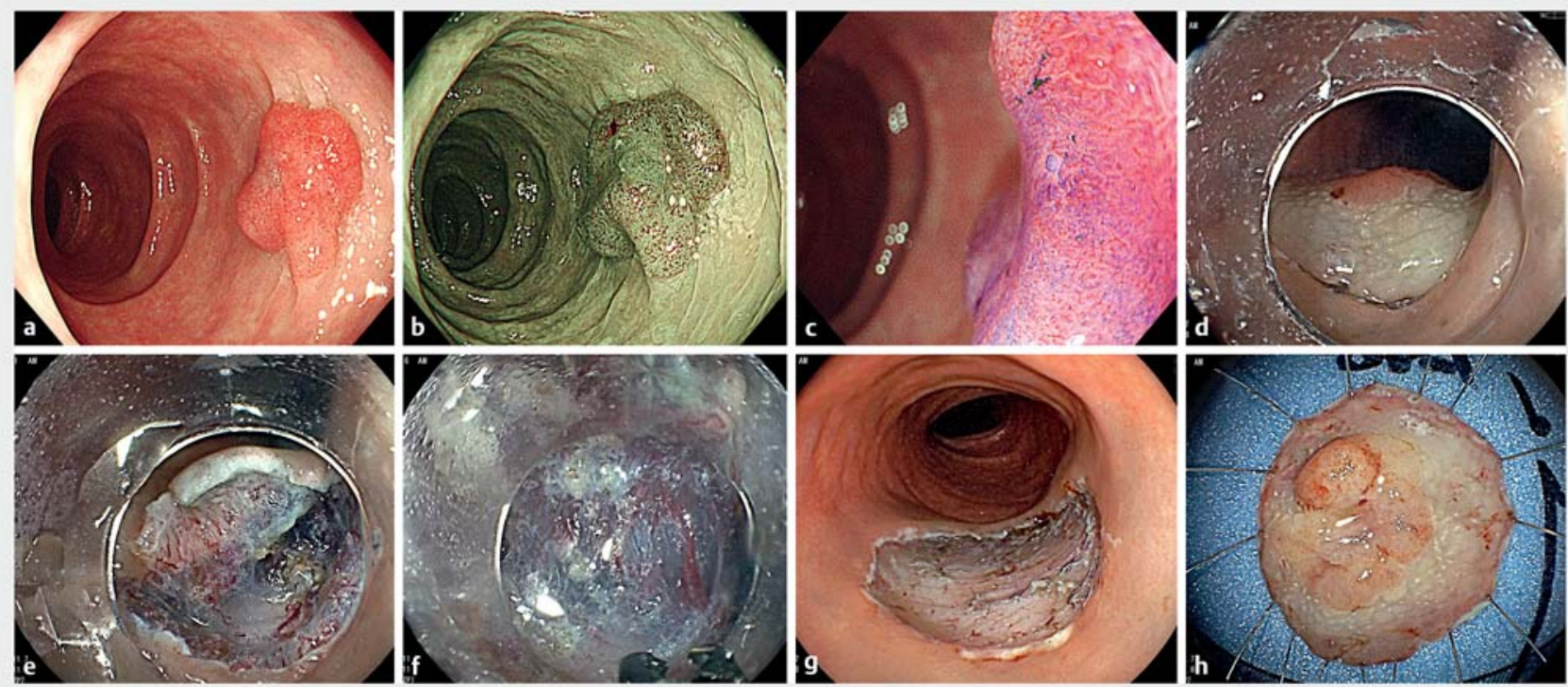

- Fig. 2 Colorectal ESD with a Clutch Cutter. a A polypoid tumor measuring $20 \mathrm{~mm}$ in the sigmoid colon. b Narrow-band imaging showed a clear tumor margin. c Magnified crystal violet imaging magnification indicated an irregular surface pattern. $\mathbf{d}$ ESD was performed by an expert. A mucosal incision was made with a 3.5-mm Clutch Cutter. e A flap was made. $\mathbf{f}$ The endoscope was stabilized in the pocket and sufficient traction was achieved. Speedy and safe submucosal dissection was performed with a Clutch Cutter. $\mathbf{g}$ En bloc resection was achieved. The procedure time was 49 minutes. h Histology showed submucosal invasive cancer with a positive vertical margin (resected specimen: $34 \times 30 \mathrm{~mm}$ ).
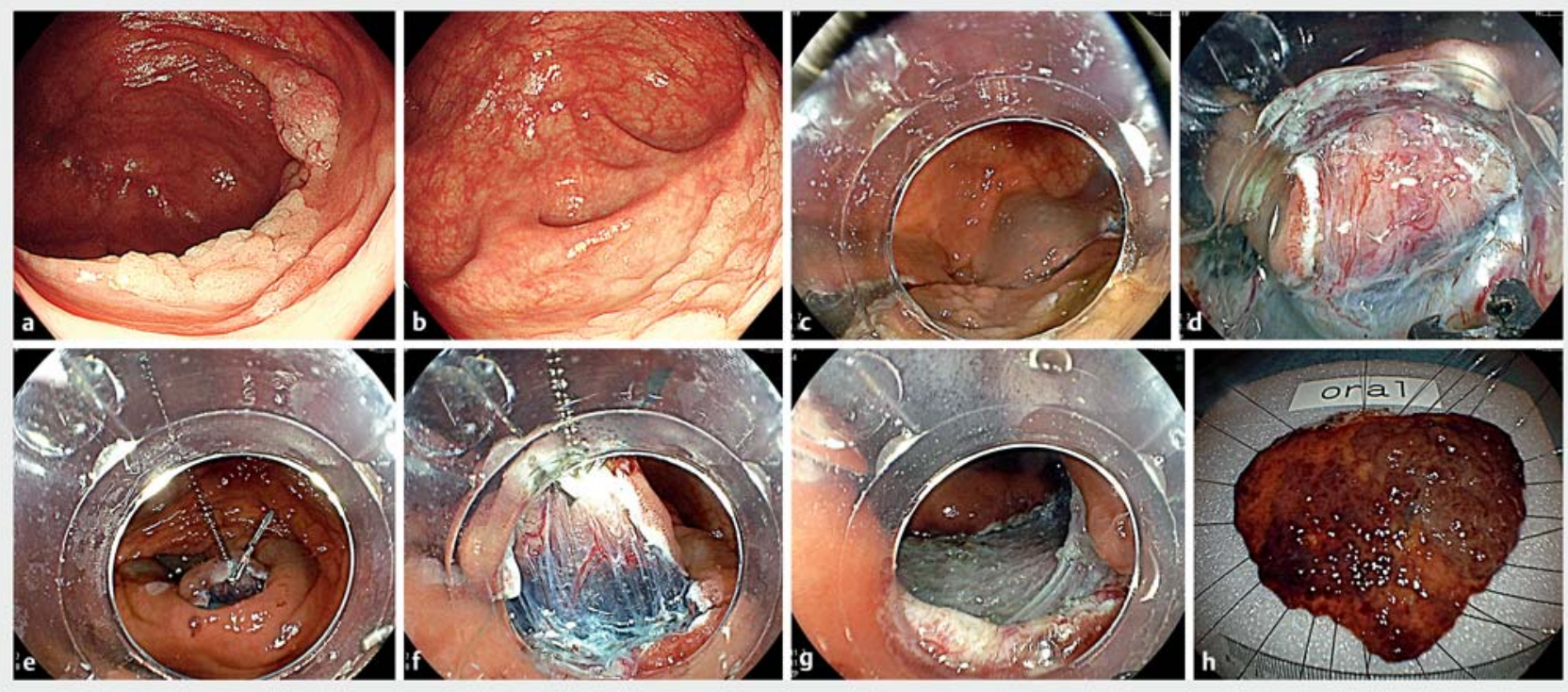

Fig. 3 Combined use of a Clutch Cutter and S-O clip in colorectal ESD. a A 62-mm non-polypoid tumor in the cecum. b The tumor did not reach the appendix. c ESD was performed by an expert. d A mucosal incision was made with 3.5-mm Clutch Cutter. e Only the center of the submucosa was dissected to make a pocket. f Sufficient traction was achieved by the S-O clip. Speedy and safe submucosal dissection was performed with the combined use of a Clutch Cutter and S-O clip.g En bloc resection was achieved. The procedure time was 112 minutes. h Histology showed tubulovillous adenoma with focal severe atypia with a negative margin (resected specimen: $72 \times 59 \mathrm{~mm}$ ).

\section{ESD procedure using the Flush knife supported by the CC}

ESD procedures under sedation were performed using ECL600ZP or ECL600ZP7 colonoscopes, the LASEREO endoscopic system, Elastic Touch, sodium hyaluronate and VIO300
$D$, just as with ESD using the CC. The method has already been described in detail [12]. In brief, a mucosal incision was performed in Endocut I mode (Effect 2, duration 2, interval 3) from the anal side of the endoscope with partial submucosal dissection. Submucosal dissection was performed in the swift 


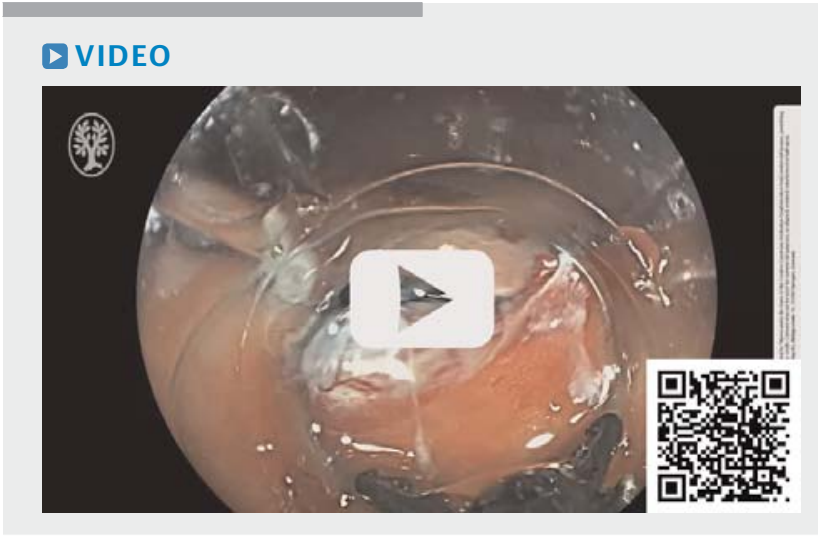

- Video 1 Colorectal endoscopic submucosal dissection with a Clutch Cutter and S-O clip for a 62-mm lesion in the cecum. Although this case was included in $\mathrm{SO}+\mathrm{CC}$ group, this case was not included after a propensity score-matching analysis ( $\triangleright$ Table 1).

coagulation mode (Effect 2, $50 \mathrm{~W}$ ). Oozing or spurting bleeding was stopped using the CC with soft coagulation mode (Effect 4, $100 \mathrm{~W})$.

\section{Statistical analyses}

The chi-square test, unpaired $t$-test and Mann-Whitney U-test were used to compare categorical variables. The unpaired $t$ test and Mann-Whitney U-test were used to compare continuous variables. $P<0.05$ was considered statistically significant. All statistical analyses were performed using the SPSS software program (version 22.0 for Windows; IBM Japan, Ltd., Tokyo, Ja- pan). In Studies 1 and 2, we performed a propensity scorematching analysis between the two groups to reduce the effects of any selection bias for endoscopic devices. Propensity scores were estimated using a logistic regression model with the following covariates: patient age and sex, tumor size, macroscopic type, tumor location and ESD operator. The one-toone matched pairs between the two groups were individually obtained by matching subjects on the logit of the propensity score using nearest-neighbor matching within a caliper width of 0.01 . In addition, we used absolute standardized difference (ASD) as a balance diagnostic and evaluated ASD $<0.2$ as well balanced.

\section{Results}

\section{Study 1: SO vs. CC}

A total of 125 patients with colorectal tumor were included in this study: 60 patients who underwent ESD in the SO group and 65 patients who underwent ESD in the CC group. The study flowchart and distribution of propensity scores are shown (^Fig.1). After propensity score-matching, there were 36 matched pairs of patients between the two groups.

Baseline characteristics of patients with colorectal tumor in the SO and CC groups are summarized in - Table 1. Before matching, the age, gender ratio, tumor location and estimated tumor size were not significantly different; however, the tumor morphology and physician experience were significantly different between the two groups. In 36 the pairs of propensity score-matched patients, the baseline characteristics of the two groups were comparable.

\begin{tabular}{|c|c|c|c|c|c|c|c|c|}
\hline & \multicolumn{2}{|l|}{ Before matching } & \multirow[t]{2}{*}{ ASD } & \multirow{2}{*}{$\begin{array}{l}P \\
\text { value }\end{array}$} & \multicolumn{2}{|l|}{ After matching } & \multirow[t]{2}{*}{ ASD } & \multirow{2}{*}{$\begin{array}{l}P \\
\text { value }\end{array}$} \\
\hline & $S O+C C(n=60)$ & $C C(n=65)$ & & & $\mathrm{SO}+\mathrm{CC}(\mathrm{n}=36)$ & $\mathrm{CC}(n=36)$ & & \\
\hline Age (years), median (range) & $71(44-88)$ & $69(41-87)$ & 0.079 & 0.661 & $71(44-84)$ & $71(41-85)$ & 0.173 & 0.470 \\
\hline Sex, male/female & $36 / 24$ & $41 / 24$ & 0.063 & 0.724 & $21 / 15$ & $25 / 11$ & 0.232 & 0.326 \\
\hline Tumor location, n (\%) & & & 0.185 & 0.118 & & & 0.039 & 0.946 \\
\hline - Right colon & $43(71.6)$ & $38(58.4)$ & & & $26(72.2)$ & $25(69.4)$ & & \\
\hline - Left colon & $10(16.7)$ & $10(15.4)$ & & & $5(13.9)$ & $5(13.9)$ & & \\
\hline - Rectum & $7(11.7)$ & $17(26.2)$ & & & $5(13.9)$ & $6(16.7)$ & & \\
\hline Morphology, n (\%) & & & 0.499 & 0.0072 & & & $<0.001$ & $>0.999$ \\
\hline - Non-polypoid & $54(0.90)$ & $46(70.8)$ & & & $32(88.9)$ & 32 (88.9) & & \\
\hline - Polypoid & $6(0.10)$ & $19(29.2)$ & & & $4(11.1)$ & $4(11.1)$ & & \\
\hline $\begin{array}{l}\text { - Median estimated } \\
\text { tumor size (range, mm) }\end{array}$ & $25(10-80)$ & $20(10-40)$ & 0.556 & 0.0022 & $20(10-42)$ & $20(10-40)$ & 0.048 & 0.842 \\
\hline Operator, n (\%) & & & 0.422 & 0.0196 & & & 0.116 & 0.624 \\
\hline - More experienced & $41(68.3)$ & $31(47.7)$ & & & $22(61.1)$ & $24(66.7)$ & & \\
\hline - Less-experienced & $19(31.7)$ & $34(52.3)$ & & & $14(38.9)$ & $12(33.3)$ & & \\
\hline
\end{tabular}


The clinicopathological features of resected colorectal tumors in the SO group and CC group are summarized in $>$ Table 2. The histology, median resected tumor size, median resected specimen size, and ratio of severe fibrosis were not significantly different between the two groups. The median ESD specimen sizes were 30 and $33 \mathrm{~mm}$ in the SO and CC groups, respectively, with no significant difference $(P=0.783)$.

The technical outcomes of ESD for the SO group and CC group are summarized in $>$ Table $\mathbf{3}$. In total cases as well as in those treated by an experienced operator, the median procedure time in the SO group was not significantly shorter than that in the CC group (Total cases, 40 and 42 minutes, $P=$ 0.626 ; and more experienced operator, 38 and 36 minutes, $P=$ $0.537)$. However, in the less-experienced endoscopist with more experienced operator cases, the median procedure time in the SO group was significantly shorter than that in the CC group (Less-experienced endoscopist with more experienced operator, 42 and 65 minutes, $P=0.037$ ). The median time for the retrieval of the $\mathrm{S}-\mathrm{O}$ clip in the total cases of the $\mathrm{SO}$ group was 4 minutes, in the cases treated by less-experienced endoscopist with more experienced operator of the SO group, it was 4 minutes, in the cases treated by an experienced operator, it was 3 minutes. The en bloc resection and R0 resection rates were $97.2 \%$ and $88.9 \%$ for the SO group and $100 \%$ and $91.7 \%$ for the CC group, respectively. The self-completion rate and semi-self completion rate in the SO group among less-experienced endoscopists were not significantly different compared with in the CC group (self-completion rate: $14.3 \%$ vs. $16.7 \%, P$ $=0.867$, semi-completion rate: $50.0 \%$ vs. $50.0 \%, P=1.000$ ) ( $\triangleright$ Table 3). Perforation during the procedure occurred in one case only, in the SO group. There were no cases of delayed bleeding in either of these two groups.

- Table2 Clinicopathological features of resected colorectal tumors in Study 1 (SO + CC vs. CC).

\begin{tabular}{|c|c|c|c|}
\hline & $\begin{array}{l}\text { So }+C C \\
(n=36)\end{array}$ & $\begin{array}{l}\text { CC } \\
(n=36)\end{array}$ & $P$ value \\
\hline Histology, n (\%) & & & 0.307 \\
\hline - Serrated lesions & $7(19.4)$ & $2(5.6)$ & \\
\hline - Adenoma & $19(52.9)$ & $23(63.8)$ & \\
\hline - Intramucosal cancer & $7(19.4)$ & $9(25.0)$ & \\
\hline - Adenocarcinoma ( $\geq \mathrm{sm})$ & $3(8.3)$ & $2(5.6)$ & \\
\hline Median resected tumor size (range, $\mathrm{mm}$ ) & $23(10-45)$ & $20(10-40)$ & 0.390 \\
\hline Median resected specimen size (range, $\mathrm{mm}$ ) & $30(20-50)$ & $33(22-50)$ & 0.783 \\
\hline Severe fibrosis status, n (\%) & $5(13.9)$ & $1(2.8)$ & 0.088 \\
\hline
\end{tabular}

- Table 3 Technical outcomes of ESD in Study 1 (SO + CC vs. CC).

\begin{tabular}{|c|c|c|c|}
\hline & $\begin{array}{l}\mathrm{SO}+\mathrm{CC} \\
(n=36)\end{array}$ & $\begin{array}{l}\text { CC } \\
(n=36)\end{array}$ & $P$ value \\
\hline \multicolumn{4}{|l|}{ Median procedure time (range, min) } \\
\hline - All cases (range, min) & $40(16-118)$ & $42(20-79)$ & 0.626 \\
\hline - Experts (range, min) & $38(16-118)$ & $36(20-76)$ & 0.537 \\
\hline - Less-experienced endoscopists with experts (range, min) & $42(27-69)$ & $65(30-79)$ & 0.036 \\
\hline Self-completion rate of less-experienced endoscopists, n (\%) & $2(14.3)$ & $2(16.7)$ & 0.867 \\
\hline Semi-self-completion rate of less-experienced endoscopists, $n$ (\%) & $7(50.0)$ & $6(50.0)$ & 1.000 \\
\hline En bloc resection, $\mathrm{n}(\%)$ & $35(97.2)$ & $36(100)$ & 0.314 \\
\hline Complete resection (R0), $\mathrm{n}(\%)$ & $32(88.9)$ & $33(91.7)$ & 0.691 \\
\hline \multicolumn{4}{|l|}{ Adverse events } \\
\hline - Perforation, n (\%) & $1(2.8)$ & $0(0)$ & 0.315 \\
\hline - Delayed bleeding, n (\%) & $0(0)$ & $0(0)$ & \\
\hline
\end{tabular}




\section{Study 2: CC vs. Flush}

A total of 185 patients with colorectal tumor were included in this study: 65 patients who underwent ESD in the CC group and 120 patients who underwent ESD in the Flush group. The study flowchart and distribution of propensity scores are shown ( Fig.1). After propensity score-matching, there were 49 matched pairs of patients between the TWO groups.

Baseline characteristics of patients with colorectal tumor in the CC and Flush groups are summarized in $>$ Table 4 . Before matching, the age, gender ratio, tumor location and tumor morphology were not significantly different; however, the estimated tumor size and physician experience were significantly different between the two groups. In the 49 pairs of propensity score-matched patients, the baseline characteristics of the two groups were comparable.

The clinicopathological features of resected colorectal tumors in the CC group and Flush group are summarized in $>\mathbf{T a}$ ble 5 . The histology, median resected tumor size, median resected specimen size and ratio of severe fibrosis were not significantly different between the two groups. The median ESD specimen sizes were 32 and $35 \mathrm{~mm}$ in the CC and Flush groups, respectively, with no significant difference $(P=0.325)$.

The technical outcomes of ESD for the CC group and Flush group are summarized in $>$ Table 6 . In total cases as well as in those treated by an experienced operator, the median procedure time in the CC group was not significantly shorter than that in the Flush group (Total cases, 45 and 53 minutes, $P=$

- Table 4 Baseline characteristics of all patients before and after matching in Study 2 (CC vs. Flush).

\begin{tabular}{|c|c|c|c|c|c|c|c|c|}
\hline & \multicolumn{2}{|c|}{ Before matching } & \multirow[t]{2}{*}{ ASD } & \multirow[t]{2}{*}{$P$ value } & \multicolumn{2}{|c|}{ After matching } & \multirow[t]{2}{*}{ ASD } & \multirow[t]{2}{*}{$P$ value } \\
\hline & $C C(n=65)$ & Flush $(n=120)$ & & & $C C(n=49)$ & Flush $(n=49)$ & & \\
\hline Age (years), median (range) & $69(41-87)$ & $69(35-91)$ & 0.013 & 0.931 & $69(41-85)$ & $71(49-90)$ & 0.053 & 0.799 \\
\hline Sex, male/female & $41 / 24$ & $66 / 54$ & 0.165 & 0.288 & $27 / 22$ & $31 / 18$ & 0.166 & 0.676 \\
\hline Tumor location, n (\%) & & & 0.075 & 0.592 & & & 0.165 & 0.263 \\
\hline - Right colon & $38(58.4)$ & $62(51.7)$ & & & $28(57.2)$ & $30(61.2)$ & & \\
\hline - Left colon & $10(15.4)$ & $25(20.8)$ & & & $8(16.3)$ & $12(24.5)$ & & \\
\hline - Rectum & $17(26.2)$ & $33(27.5)$ & & & $13(26.5)$ & $7(14.3)$ & & \\
\hline Morphology, n (\%) & & & 0.174 & 0.252 & & & 0.045 & 0.825 \\
\hline - Non-polypoid type & $46(70.8)$ & $94(78.3)$ & & & $34(69.4)$ & $35(71.4)$ & & \\
\hline - Polypoid type & $19(29.2)$ & $26(21.7)$ & & & $15(30.6)$ & $14(28.6)$ & & \\
\hline $\begin{array}{l}\text { Median estimated tumor } \\
\text { size (range, } \mathrm{mm} \text { ) }\end{array}$ & $30(10-100)$ & $20(10-40)$ & 0.769 & $<0.0001$ & $25(15-40)$ & $25(12-50)$ & 0.019 & 0.927 \\
\hline Operator, n (\%) & & & 0.386 & 0.012 & & & 0.163 & 0.653 \\
\hline - More experienced & $31(47.7)$ & $80(66.7)$ & & & $23(46.9)$ & $22(44.9)$ & & \\
\hline - Less-experienced & $34(52.3)$ & $40(33.3)$ & & & $26(53.1)$ & $27(55.1)$ & & \\
\hline
\end{tabular}

- Table 5 Clinicopathological features of resected colorectal tumors in Study 2 (CC vs. Flush).

\begin{tabular}{|c|c|c|c|}
\hline & $\begin{array}{l}\text { CC } \\
(n=49)\end{array}$ & $\begin{array}{l}\text { Flush } \\
(n=49)\end{array}$ & $P$ value \\
\hline Histology, n (\%) & & & 0.590 \\
\hline - Serrated lesions & $6(12.2)$ & $3(6.1)$ & \\
\hline - Adenoma-intramucosal carcinoma & $41(69.4)$ & $37(89.8)$ & \\
\hline - Adenocarcinoma ( $\geq \mathrm{sm}$ ) & $9(18.4)$ & $2(4.1)$ & \\
\hline Median resected tumor size (range, $\mathrm{mm}$ ) & $23(15-40)$ & $25(10-60)$ & 0.187 \\
\hline Median resected specimen size (range, $\mathrm{mm}$ ) & $32(22-50)$ & $35(15-68)$ & 0.325 \\
\hline Severe fibrosis status, $\mathrm{n}(\%)$ & $2(4.1)$ & $3(6.1)$ & 0.630 \\
\hline
\end{tabular}


- Table 6 Technical outcomes of ESD in Study 2 (CC vs. Flush).

\begin{tabular}{|l|l|l|}
\hline & CC & $\begin{array}{l}\text { Flush } \\
\text { (n=49) }\end{array}$ \\
\hline Median procedure time (range, min) & & \\
\hline - All cases (range, min) & $45(15-83)$ & $53(20-224)$ \\
\hline - Experts (range, min) & $36(15-76)$ & $35(20-224)$ \\
\hline - Less-experienced endoscopists with experts (range, min) & $52(29-83)$ & $67(23-160)$ \\
\hline Self-completion rate of less-experienced endoscopists, $n$ (\%) & $6(23.1)$ & $1(3.7)$ \\
\hline Semi-self-completion rate of less-experienced endoscopists, $n(\%)$ & $2(7.4)$ \\
\hline En bloc resection, $n$ (\%) & $14(53.8)$ & $48(98.0)$ \\
\hline Complete resection (R0), $n$ (\%) & $49(100)$ & $46(95.8)$ \\
\hline Adverse events & $46(95.8)$ & 0.232 \\
\hline - Perforation, $n$ (\%) & & 0.038 \\
\hline - Delayed bleeding, $n$ (\%) & $0(0)$ & $1(2.0)$ \\
\hline CC, Clutch Cutter; Flush, Flush knife. & $0(0)$ & 0.0002 \\
\hline
\end{tabular}

0.589; and more experienced operator, 36 and 35 minutes, $P=$ 0.232 ). However, in the less-experienced endoscopist with more experienced operator cases, the median procedure time in the CC group was significantly shorter than that in the Flush group (Less-experienced endoscopist with more experienced operator, 52 and 67 minutes, $P=0.038$ ). The en bloc resection and $\mathrm{R} 0$ resection rates were $100 \%$ and $93.9 \%$ for the CC group and $98 \%$ and $93.9 \%$ for the Flush group, respectively. The selfcompletion rate in the CC group among less-experienced endoscopists was significantly higher than in the Flush group (23.1\% vs. $3.7 \%, P=0.038$ ) ( $>$ Table 6 ). The semi-self-completion rate in the CC group among less-experienced endoscopists was significantly higher than in the Flush group $(53.8 \%$ vs. $7.4 \%, P=0.0002$ ) ( $>$ Table 6 ). Perforation during the procedure occurred in one case only, in the Flush group. There were no cases of delayed bleeding in either of these two groups.

\section{Discussion}

In this study, we demonstrated the efficacy of using the CC and S-O clip during colorectal ESD. The results of Study 1 showed that the combined use of the S-O clip and CC was associated with a significant reduction in the procedure time for less-experienced endoscopists compared with the CC alone. Furthermore, the results of Study 2 showed that the use of the CC was associated with a significant reduction in the procedure time for less-experienced endoscopists compared with a needletype knife.

A previous study showed that the use of the CC for colorectal ESD performed by one endoscopist with prior experience of only seven cases of conventional ESD was not associated with a reduction in the procedure time [4]. It was also reported that the use of other scissor-type knives for colorectal ESD performed by two endoscopists with prior experience of about 50 cases of conventional gastric ESD was not associated with a re- duction in the procedure time [9]. However, for gastric ESD, it was reported that the use of the CC was associated with a significant reduction in the procedure time for less-experienced endoscopists compared with a blade-type knife [7]. Furthermore, a previous randomized controlled trial (RCT) showed that the use of the CC was associated with a significant increase in the procedure time compared with the use of the IT knife 2 [15].

In Study 2, the CC and needle-type knife were compared, which has not been attempted before. We found that the procedure time for colorectal ESD was significantly shorter among less-experienced endoscopists in the CC group than among those in the Flush group. The duration of procedures using scissor-type knives among only less-experienced endoscopists may have been shortened in the present study for several reasons. Regarding the merits of CC compared to Flush knife, CC enabled to coagulate vessels and stop unexpected bleeding. CC prevented the use of hemostatic forceps and it saved procedure time partially. In addition, CC could be used by less-experienced endoscopists in a difficult situation such as paradoxical movement and breathing movement. Because a tissue can be dissected by CC after catching a tissue by CC so that there were theoretically no unintentional perforations. This also resulted in the decrease of procedure time. On the other hand, Flush knife could not stop bleeding especially in less-experienced endoscopists. Additionally, it could not be used in these difficult situations because of possibility of perforation due to unintentional movement of knife. Actually, in Study 2, the selfcompletion and semi- self-completion rates in the CC group were significantly higher than those in the Flush group. In the Flush group, less-experienced endoscopists were changed to supervisors in the following cases: the estimated procedure time for mucosal incision and submucosal dissection exceeded 60 minutes and there were uncontrollable hemorrhaging or perforation. On the other hand, in the CC group, less-experi- 
enced endoscopists were changed to supervisors only in the following cases: the estimated procedure time for mucosal incision and submucosal dissection was expected to exceed 60 minutes.

A previous RCT showed that the mean procedure time for colorectal ESD was significantly shorter with the S-O clip than without the S-O clip; however, the authors in that study used only needle-type knives [10]. In Study 1, we found that the combined use of the S-O clip and CC for colorectal ESD reduced the procedure time significantly among less-experienced endoscopists compared with the use of the CC alone. No previous reports have made such a comparison. This finding seems plausible, as the S-O clip allowed us to obtain better visualization and perform submucosal dissection easily. A previous RCT showed that the mean procedure time for esophageal and colorectal ESD was significantly shorter with traction, such as via the clip-and-thread technique, than without traction, and they used both needle-type and blade-type knives for ESD [16]. These results suggest that the combined use of CC with traction also allowed colorectal ESD to be completed more quickly than without traction. This finding may be useful for colorectal ESD, especially that performed by less-experienced endoscopists.

In the present study, perforation during ESD occurred in only one case both in the Flush group and the SO group. All the cases of perforation were endoscopically sutured and perforation during ESD may no longer be a serious problem. We had no delayed bleeding. It was reported that a scissor-type knife might reduce the risk of perforation $[2,9,17]$. In a retrospective cohort study, the number of intraoperative bleeding points were significantly fewer in the CC group than in the conventional device group [7]. The CC can safely grasp, retract, dissect, and coagulate target tissues in a similar fashion to the way biopsy forceps are used; therefore, we encountered no unexpected incisions $[1,7]$.

Skills and experience of assistants are as important as the skills of endoscopists when CC is used during ESD. In all cases in the $\mathrm{SO}+\mathrm{CC}$ and $\mathrm{CC}$ groups, CC were rotated by the same three assistants. In our center, less-experienced assistants and endoscopists were trained with a hands-on model regularly before clinical cases. They also referred to a document and a leaflet that showed how to use CC correctly [18]. For assistants, they can almost master the handling of CC by assisting in about 10 cases. Thus, we thought the influence of the learning curve was small in each group.

The cost of the devices varies in each country. We showed the cost of each group in Japan. The average medical cost for ESD -devices was $¥ 48,000$ for the SO + CC group (S-O clip, Clutch Cutter), $¥ 43,000$ for the CC group (Clutch Cutter), and $¥ 44,800$ for the Flush group (Flush knife BT-S, Coagrasper). The medical costs for ESD devices in $\mathrm{SO}+\mathrm{CC}$ group was more than in the CC or Flush groups. SO + CC showed benefits in terms of a shorter ESD time for less-experienced endoscopists in our study, so SO + CC may have benefits in terms of the costs for the injection solution and the labor costs for the medical staffs. $\mathrm{SO}+\mathrm{CC}$ or CC using ESD is expected to be almost equally costeffective with Flush using ESD.
Several limitations of this study should be acknowledged. Our study was a retrospective, single-center study and had a small sample size. We had already included many of the same patients in other studies, but the purpose of those previous studies was different. Substantial heterogeneity of the operators and different timeline in each group may lead to bias. In addition, the learning effect in the combined use of the S-O clip and CC compared to the CC might affect the results although propensity score-matching was performed to prevent selection bias. Thus, further RCTs including large samples should be performed to evaluate the efficacy of the CC and SO clip.

\section{Conclusions}

In summary, we performed a retrospective study to examine the efficacy of CC and combined use of the S-O clip and CC for colorectal ESD. We showed for the first time that, for less-experienced endoscopists, CC was associated with a shorter procedure time and that combined use of CC and S-O clip was also associated with a significant reduction in procedure time. Therefore, combined use of CC and S-O clip is recommended for colorectal ESD by less-experienced endoscopists.

\section{Competing interests}

Drs. Yoshida (J162001222) and Naito (J162003400, J162003294) received a research grant from Fujifilm Medical Co., Ltd. Dr. Itoh (J082003006) was affiliated with a department that was partially funded by Fujifilm Medical Co., Ltd.

\section{References}

[1] Akahoshi K, Okamoto R, Akahane H et al. Endoscopic submucosal dissection of early colorectal tumors using a grasping-type scissors forceps: a preliminary clinical study. Endoscopy 2010; 42: 419-422

[2] Homma K, Otaki Y, Sugawara M et al. Efficacy of novel SB knife Jr examined in a multicenter study on colorectal endoscopic submucosal dissection. Dig Endosc 2012; 24: 117-120

[3] Akahoshi K, Akahane H, Motomura Y et al. A new approach: endoscopic submucosal dissection using the Clutch Cutter(R) for early stage digestive tract tumors. Digestion 2012; 85: 80-84

[4] Akahoshi K, Akahane H. A new breakthrough: ESD using a newly developed grasping type scissor forceps for early gastrointestinal tract neoplasms. World J Gastrointest Endosc 2010; 2: 90-96

[5] Akahoshi K, Kubokawa M, Gibo J et al. Endoscopic submucosal dissection of gastric adenomas using the clutch cutter. World J Gastrointest Endosc 2017; 9: 334-340

[6] Otsuka Y, Akahoshi K, Yasunaga K et al. Clinical outcomes of Clutch Cutter endoscopic submucosal dissection for older patients with early gastric cancer. World J Gastrointest Oncol 2017; 9: 416-422

[7] Dohi O, Yoshida N, Terasaki K et al. Efficacy of clutch cutter for standardizing endoscopic submucosal dissection for early gastric cancer: a propensity score-matched analysis. Digestion 2018: doi:10.1159/ 000495287

[8] Sawas T, Visrodia KH, Zakko L et al. Clutch cutter is a safe device for performing endoscopic submucosal dissection of superficial esopha- 
geal neoplasms: a western experience. Dis Esophagus 2018; 31: doi:10.1093/dote/doy054

[9] Yamashina T, Takeuchi Y, Nagai K et al. Scissor-type knife significantly improves self-completion rate of colorectal endoscopic submucosal dissection: Single-center prospective randomized trial. Dig Endosc 2017; 29: 322-329

[10] Ritsuno H, Sakamoto N, Osada T et al. Prospective clinical trial of traction device-assisted endoscopic submucosal dissection of large superficial colorectal tumors using the S-O clip. Surg Endosc 2014; 28: 3143-3149

[11] Sakamoto N, Osada T, Shibuya T et al. Endoscopic submucosal dissection of large colorectal tumors by using a novel spring-action S-O clip for traction (with video). Gastrointest Endosc 2009; 69: 13701374

[12] Yoshida N, Naito Y, Murakami T et al. Tips for safety in endoscopic submucosal dissection for colorectal tumors. Ann Transl Med 2017; 5: 185

[13] Yoshida N, Naito Y, Murakami T et al. Safety and efficacy of a sameday low-volume $1 \mathrm{~L}$ PEG bowel preparation in colonoscopy for the el- derly people and people with renal dysfunction. Dig Dis Sci 2016; 61 : 3229-3235

[14] Matsumura S, Yoshida N, Inoue K. Colorectal endoscopic submucosal dissection with a scissor-type knife and a traction device. Dig Endosc 2019: doi:10.1111/den.13344

[15] Nagai K, Uedo N, Yamashina T et al. A comparative study of graspingtype scissors forceps and insulated-tip knife for endoscopic submucosal dissection of early gastric cancer: a randomized controlled trial. Endosc Int Open 2016; 4: E654-E660

[16] Yamasaki Y, Takeuchi Y, Uedo $N$ et al. Efficacy of traction-assisted colorectal endoscopic submucosal dissection using a clip-and-thread technique: A prospective randomized study. Dig Endosc 2018; 30: 467-476

[17] Nawata Y, Homma K, Suzuki Y. Retrospective study of technical aspects and complications of endoscopic submucosal dissection for large superficial colorectal tumors. Dig Endosc 2014; 26: 552-555

[18] Yoshida N, Dohi O, Inoue K et al. Efficacy of scissor-type knives for endoscopic mucosal dissection of superficial gastrointestinal neoplasms. Dig Endosc 2020; 32: 4-15 